\title{
Etude de la photodégradation du reactive black 5 et du reactive orange 16 en solution aqueuse en utilisant des couches minces de $\mathrm{TiO}_{2}$
}

\author{
T. KODOM ${ }^{1,2}$, G. DJANEYE-BOUNDJOU ${ }^{1 *}$, L. M. BAWA ${ }^{1}$, B. GOMBERT ${ }^{3}$ et \\ N. ALONSO-VANTE ${ }^{2}$ \\ ${ }^{1}$ Laboratoire de Chimie des Eaux, Faculté des Sciences, Université de Lomé, Boulevard Eyadema, Lomé, Togo. \\ ${ }^{2}$ Laboratoire d'Electrocatalyse, UMR-CNRS 6503, Université de Poitiers, 86022, Poitiers, France. \\ ${ }^{3}$ Laboratoire de Chimie et Microbiologie de l'Eau, UMR-CNRS 6008, Université de Poitiers, France. \\ *Auteur correspondant ; E-mail: adjaneye@yahoo.fr; Tel: 002289116581
}

\section{RESUME}

Cette étude a eu pour but l'optimisation des conditions opératoires pour l'élimination par photodégradation des colorants organiques dans les eaux. Les colorants Reactive orange 16 (RO16) et Reactive Black 5 (RB5) ont servi de molécules modèles. Leur photodégradation par le dioxyde de titane (P25) sous forme de couches minces déposées par électrophorèse sur de l'acier inox a été étudiée. La spectrophotométrie (ultra violet-visible) a été utilisée pour le suivi de la décoloration de la solution réactionnelle au cours du temps. Le modèle de Langmuir-Hinshelwood a permis de déterminer la constante apparente du premier ordre $\left(k_{a p p}\right.$, $\left.\mathrm{min}^{-1}\right)$ et la vitesse initiale $\left(r_{o}, \mathrm{~mol} / \mathrm{L} / \mathrm{min}\right)$ de dégradation. Un abattement de l'ordre de $43,8 \%$ et $28,2 \%$ respectivement pour le RB5 et le RO16 a été obtenu au bout de 120 minutes de temps de contact. La photodégradation des colorants étudiés est influencée par les facteurs tels que la concentration initiale en colorant, le $\mathrm{pH}$ et le peroxyde d'hydrogène. Le domaine de $\mathrm{pH}$ allant de 2,1 au pH libre de la solution a été très favorable à une bonne décoloration des colorants. L'ajout de $\mathrm{H}_{2} \mathrm{O}_{2}$ à la solution réactionnelle, correspondant au domaine de concentrations compris entre $0,74 \times 10^{-2} \mathrm{~mol} / \mathrm{L}$ et $7,4 \times 10^{-2} \mathrm{~mol} / \mathrm{L}$, a montré l'existence d'une concentration optimale égale à $3,7 \times 10^{-2} \mathrm{~mol} / \mathrm{L}$ pour RB5 et $5,18 \times 10^{-2} \mathrm{~mol} / \mathrm{L}$ pour RO16. Pour tous les paramètres étudiés, la photooxydation du RO16 a été plus faible que celle du RB5.

(C) 2011 International Formulae Group. All rights reserved.

Mots clés : $\mathrm{TiO}_{2}$ (P25 Degussa), Couches minces, modèle de Langmuir-Hinshelwood, Photodégradation

\section{INTRODUCTION}

Les ressources en eau de notre planète sont aujourd'hui menacées par le déversement anarchique ou non contrôlé d'effluents liquides et solides provenant des activités anthropiques. Les menaces sont amplifiées par le développement des industries et l'accroissement de la population (Chen et al., 2003). Environ $10^{7} \mathrm{~kg}$ de colorants sont utilisés par an dans le textile à travers le monde (Wong et al., 2004; Zainal et al., 2009). Cette utilisation massive de colorants menace dangereusement la qualité des eaux (Houas et al., 2001 ; Konstantinou et Albanis, 2004 ). Les effluents textiles nécessitent un traitement avant leur rejet. Les techniques d'adsorption sur charbon actif, d'ultrafiltration, d'osmose inverse ou de la coagulation par des agents 
chimiques peuvent être utilisées pour l'élimination des colorants (Galindo et al., 2001 ; Behnajady et Modirshahla, 2006; Shukla et al., 2009). Ces techniques onéreuses n'éliminent pas définitivement les composés (Galindo et al., 2001; Shukla et al., 2009). Avec ces techniques, il est nécessaire de procéder à la régénération des matériaux adsorbants qui est une étape coûteuse (Galindo et al., 2001).

Depuis quelques décennies, les techniques d'oxydation avancées constituent l'une des voies efficaces permettant la destruction totale des composés organiques. La photocatalyse a fait l'objet de nombreuses études et reste très prometteuse par sa capacité à détruire les composés organiques en molécules simples comme $\mathrm{CO}_{2}, \mathrm{H}_{2} \mathrm{O}$ et les acides minéraux (Zainal et al., 2009; Shukla et al., 2009). Elle est basée sur l'absorption des photons d'énergie supérieure ou égale à la bande interdite du photocatalyseur pour générer des électrons dans la bande de conduction et des trous dans la bande de valence. Les paires électron-trou ainsi générées peuvent se recombiner ou réagir avec des composés organiques adsorbés à la surface du semi-conducteur via les processus d'oxydo-réduction (Villarreal et al., 2004). En milieu aqueux, les trous piègent les molécules d'eau qui sont transformées en radicaux hydroxyles $\mathrm{HO}^{\circ}$. Ces radicaux hydroxyles, réagissent avec les composés organiques en solution (photodégradation indirecte). Ces mêmes trous peuvent directement oxyder les molécules organiques (photodégradation directe).

Le dioxyde de titane $\left(\mathrm{TiO}_{2}\right)$ est de loin le photocatalyseur le plus utilisé à cause de certains avantages tels que sa non toxicité, son insolubilité, sa stabilité chimique, sa bonne réponse photocatalytique, son faible coût et son abondance (Zainal et al., 2009). De nombreux auteurs ont montré l'efficacité du $\mathrm{TiO}_{2}$ (P25) en poudre dans la dépollution des eaux chargées des composés organiques
(Colon et al., 2004 ; El Hajjouji et al., 2008). Cependant, il existe des difficultés dans l'utilisation de la poudre de $\mathrm{TiO}_{2}(\mathrm{P} 25)$. Ces difficultés sont relatives à la séparation du photocatalyseur et de l'eau traitée, à l'effet d'écran limitant la photo-excitation de $\mathrm{TiO}_{2}$ et au transfert de masse (Piscopo, 2002). La photocatalyse supportée peut être une alternative en vue d'éviter ces difficultés. Le dioxyde de titane, fixé sous forme de film ou de couches minces, a une faible activité par rapport à la poudre à cause de la diminution de la surface active. Toutefois, une bonne préparation de ces couches permet d'augmenter considérablement cette photoactivité (Zhu et al., 2001). L'électrophorèse est l'une des techniques les plus utilisées pour le dépôt de $\mathrm{TiO}_{2}$ sous forme de couches minces.

Ce travail présente la photoactivité des couches minces de $\mathrm{TiO}_{2}$ (P25) Degussa obtenues par électrophorèse sur de l'acier inoxydable. Ces couches minces sont utilisées en vue de la dégradation de deux colorants textiles à savoir le Reactive Orange 16 et le Reactive Black 5. Les effets des paramètres tels que la concentration initiale, le $\mathrm{pH}$, l'acide utilisé et la présence du peroxyde d'hydrogène ont été étudiés afin d'en déduire les conditions optimales d'élimination de ces composés.

\section{MATERIEL ET METHODES}

\section{Les colorants utilisés}

\section{Le Reactive Orange 16 (RO16)}

Le Reactive Orange 16 dont la formule brute est $\mathrm{C}_{20} \mathrm{H}_{19} \mathrm{~N}_{3} \mathrm{O}_{11} \mathrm{~S}_{3}, 2 \mathrm{Na}(\mathrm{M}=617,54$ g. $\mathrm{mol}^{-1}$ ) donne une coloration orange comme son nom l'indique et absorbe à la longueur d'onde maximale de $495 \mathrm{~nm}$.

\section{Le Reactive Black 5 (RB5)}

$\mathrm{La}$ formule brute de ce colorant est $\mathrm{C}_{26} \mathrm{H}_{25} \mathrm{~N}_{5} \mathrm{O}_{19} \mathrm{~S}_{6}$, 4Na $\left(\mathrm{M}=991,82\right.$ g.mol $\left.{ }^{-1}\right)$. Soluble dans l'eau, il est d'une couleur bleu sombre.

Les solutions de colorants de concentration $5 \mathrm{mgC} / \mathrm{L}$ soit $20,8 \mu \mathrm{mol} / \mathrm{L}$ pour 
le RO16 et 16,1 $\mu \mathrm{mol} / \mathrm{L}$ pour le RB5 ont été préparées avec de l'eau distillée.

\section{Réacteur}

Le montage expérimental utilisé pour la dégradation des composés étudiés comprend: une cuve en verre (réacteur), une lampe UV, une pompe et un réservoir (Figure 1).

La cuve en verre est de forme rectangulaire (44 $\mathrm{cm}$ de longueur $\times 8 \mathrm{~cm}$ de largeur $\times 6 \mathrm{~cm}$ de hauteur). La solution parvient au réacteur par une ouverture située sur une largeur et est uniformément répartie dans le réacteur par un tamis en polyéthylène placé à cette entrée. La sortie de la solution se fait par quatre ouvertures circulaires de 1 $\mathrm{cm}$ de diamètre. Au dessus du réacteur en verre est placé un réflecteur en acier inoxydable $(45 \mathrm{~cm} \times 9 \mathrm{~cm} \times 9 \mathrm{~cm})$ permettant l'isolement des rayonnements UV. Le réacteur et le réflecteur sont couverts d'un ruban noir ou d'un carton bien épais afin d'éviter l'influence d'une lumière extérieure et d'éliminer toute fuite de la lumière UV. La lampe Black Light Blue F15T8/BL d'une puissance de 15 Watts dont le tube fait $40 \mathrm{~cm}$ de long a été utilisée pour les expériences de photodégradation. C'est une lampe dont la radiation maximale est de $365 \mathrm{~nm}$ et elle a été placée au dessus du fond de la cuve. Elle a été allumée 30 minutes avant le début des tests de photocatalyse. Le réservoir est un cylindre en verre constitué d'une double paroi permettant la régulation de la température grâce à une circulation d'eau. La pompe submersible de type NEW-JET800 est introduite dans le réservoir rempli de la solution du composé organique à dégrader. Cette pompe a permis la circulation de la solution réactionnelle entre le réacteur et le réservoir. Elle refoule la solution réactionnelle du réservoir vers le réacteur par le biais des tuyaux transparents en polychlorure de vinyle (PVC) avec un débit de $180 \mathrm{~L} / \mathrm{h}$.

\section{Suivi de la décoloration des solutions réactionnelles}

La dégradation du Reactive Black 5 et du Reactive Orange 16 est suivie par une mesure de l'absorbance à leur longueur d'onde d'absorption maximale à savoir $\lambda_{\max }=$ $599 \mathrm{~nm}$ pour RB5 et $\lambda_{\max }=495 \mathrm{~nm}$ pour RO16. Le spectrophotomètre UV-Visible Genesys $10 \mathrm{UV}-\mathrm{Vis}$ a été utilisé pour la mesure de l'absorbance. Des expériences portant sur le suivi de la cinétique de décoloration ont été répétées trois fois dans chaque cas. Dans le cas de l'étude du vieillissement, pour chaque colorant étudié, quatre tests ont été réalisés avec une plaque de couche mince de $\mathrm{TiO}_{2}(\mathrm{P} 25)$.

\section{Préparation des couches de $\mathrm{TiO}_{2}$ (P25) Degussa}

Tous les produits chimiques utilisés sont de pureté analytique. L'élaboration des couches minces a été effectuée à partir de 1,53 $\mathrm{g}$ de $\mathrm{TiO}_{2} \quad(\mathrm{P} 25)$ et d'un mélange d'acéthylacétone, d'eau distillée et du Triton X 100 comme surfactant non ionique ( Pedroza et al., 2007). Le mélange final est obtenu avec du méthanol afin d'avoir une concentration de 12,5 g/L de $\mathrm{TiO}_{2}$. La Figure 2 présente le schéma du montage expérimental réalisé pour la préparation des couches minces. Toutes les couches sont préparées dans des conditions identiques en imposant une tension continue de $10 \mathrm{~V}$ entre le substrat (cathode) et une électrode en acier inoxydable. Le dépôt des films de $\mathrm{TiO}_{2}$ s'effectue sur la cathode pendant 20 secondes. Les deux électrodes sont séparées par une distance de $1 \mathrm{~cm}$. L'acier inoxydable 304L dont l'épaisseur est de $0,5 \mathrm{~mm}$ a été utilisé. Ces plaques d'acier ont une dimension de 19,9 $\mathrm{cm}$ sur $7,8 \mathrm{~cm}$. Le dépôt se fait sur une dimension de $15,4 \mathrm{~cm} \times 7,8 \mathrm{~cm}$ en limitant la surface par une bande adhésive à $4,5 \mathrm{~cm}$ d'une extrémité. Les couches obtenues ont été séchées à la température du laboratoire en quelques minutes puis traitées à $450{ }^{\circ} \mathrm{C}$ 
pendant une heure avec un four rectangulaire étanche de marque Nabertherm (30-3000 ${ }^{\circ} \mathrm{C}$ ). Les couches minces obtenues au cours de ce travail ont été caractérisées par diffraction des rayons X (DRX) et par spectroscopie Raman afin de déterminer la microstructure et les différentes phases cristallines du photocatalyseur fixé.

\section{RESULTATS}

\section{Caractérisations des couches}

La Figure 3 montre le spectre Raman obtenu à partir des couches de $\mathrm{TiO}_{2}(\mathrm{P} 25)$ sur acier inoxydable. Les pics caractéristiques suivants ont été obtenus : 142,3; 198 ; 339,9 ; 518,1 et $638,1 \mathrm{~cm}^{-1}$.

Pour avoir des informations plus complètes sur la composition des couches, la diffraction des rayons $\mathrm{X}$ a été réalisée (Figure 4). Le diffractogramme a donné deux pics majeurs aux angles $2 \theta=25,4^{\circ}$ et $2 \Theta=27,6$.

\section{Photooxydation des colorants}

De nombreux résultats des expériences portant sur la dégradation photocatalytique et utilisant le système $\mathrm{TiO}_{2}$ sous la lumière (UV ou visible) ont montré que la vitesse de dégradation des colorants suit la loi de Langmuir-Hinshelwood (Galindo et al., 2000 ; Houas et al., 2001). Ce modèle, souvent utilisé pour décrire des phénomènes relevant de la catalyse hétérogène, a été utilisé pour l'étude de la photooxydation des deux colorants.

Les valeurs des absorbances reportées dans la présente étude sont obtenues à partir de la moyenne des trois mesures des absorbances. L'écart type a varié de 0 à $5 \%$ sur les absorbances.

Des expériences ont été effectuées en vue de déterminer la part de l'élimination des colorants étudiés par adsorption sur le catalyseur et par photolyse en milieu aqueux. Les résultats des expériences comparant l'élimination des colorants de la solution réactionnelle par la photolyse, l'adsorption et la photocatalyse sont présentés sur la Figure 5.
Dans les conditions opératoires de l'étude, la photolyse et l'adsorption des colorants à l'obscurité n'ont éliminé que des fractions très faibles de RB5 et RO16. La quantité de colorants RB5 qui s'est absorbée sur les couches de $\mathrm{TiO}_{2}$ est légèrement plus importante que celle du colorant RO16 (6,3\% pour RB5 et 5,7 \% pour RO16). La photolyse n'a permis qu'un taux d'abattement des deux colorants inférieur à $1 \%$. Les taux d'abattement de la photocatalyse au bout de 120 min ont été de l'ordre $43,8 \%$ et $28,2 \%$ respectivement pour le RB5 et le RO16 (Tableau 1). Ces taux ont montré l'efficacité du système photocatalytique à dégrader les colorants textiles étudiés par rapport à la photolyse.

\section{Etude du vieillissement des couches}

L'effet du vieillissement des couches minces de $\mathrm{TiO}_{2}$ peut intervenir au cours de leur utilisation et réduire leur efficacité photodégradante. C'est pour cette raison qu'il a été envisagé dans ce travail une étude du vieillissement du matériel utilisé comme catalyseur. Pour chaque colorant étudié, quatre tests ont été réalisés avec une plaque de couches minces de $\mathrm{TiO}_{2}$ (P25) Degussa. La Figure 6 présente les constantes apparentes de vitesse $\mathrm{k}_{\mathrm{app}}$ en fonction du nombre d'essais pour une plaque. Les valeurs des constantes apparentes de vitesses ont baissé du premier au quatrième essai. Cette baisse est plus marquée dans le cas de la photodégradation du réactive Black 5. Après une quatrième utilisation, la couche mince a subi un traitement thermique par recuit à $450{ }^{\circ} \mathrm{C}$ et réutilisée en vue de déterminer l'effet du traitement thermique des couches minces sur la dégradation en solution aqueuse du RB5. La Figure 7 présente l'évolution de l'absorbance en fonction du temps pour le premier, le quatrième et le cinquième essai. La constante apparente à la première utilisation de cette couche mince traitée à $450{ }^{\circ} \mathrm{C}$ est de $3,6 \times 10^{-3} \mathrm{~min}^{-1}$. Les résultats des essais avec le 
RB5 ont montré donc un taux de reprise de $33,3 \%$ de l'activité photocatalytique après le traitement thermique.

\section{Influence de la concentration initiale du colorant}

L'influence de la concentration initiale de RB5 et RO16 a également été étudiée. Les résultats des essais effectués pour les concentrations en colorant de $2,5 \mathrm{mgC} / \mathrm{L}, 5$ $\mathrm{mgC} / \mathrm{L}$ et $10 \mathrm{mgC} / \mathrm{L}$ sont représentés sur la Figure 8. Les constantes apparentes de vitesse et les vitesses initiales de dégradation obtenues à partir de la Figure 8 sont représentées dans le Tableau 2. Les valeurs des constantes apparentes de vitesse, $\mathrm{k}_{\mathrm{app}}$, ont augmenté lorsque les concentrations en colorant diminuaient de 10 à 2,5 mgC/L. Par contre, la vitesse initiale, $\mathrm{r}_{\mathrm{o}}$, a augmenté avec la concentration.

\section{Influence du pH et de la nature de l'acide sur la photodégradation}

L'étude de l'influence du $\mathrm{pH}$ sur la photodégradation des colorants RB5 et RO16 a été aussi effectuée. Le pH initial de la solution réactionnelle a été fixé par ajout des solutions d'acide sulfurique et de soude concentrée. L'évolution de la dégradation des deux colorants en fonction du temps aux valeurs de pH étudiées a été représentée sur la Figure 9. Les constantes apparentes de vitesse ont été déduites des courbes de la Figure 9 et sont regroupées dans le Tableau 3. La constante apparente de vitesse la plus grande a été obtenue au $\mathrm{pH}$ égal à 2,1.

Les acides utilisés peuvent influencer de par leur nature l'activité du catalyseur. L'influence de la nature de l'acide utilisé pour ajuster la solution réactionnelle au $\mathrm{pH}$ désiré a été aussi étudiée. Cette étude a porté sur les acides sulfurique, nitrique et phosphorique. Les abattements obtenus au cours du temps pour les deux colorants en présence des différents acides sont représentés à la Figure 10. La meilleure dégradation des deux colorants a été obtenue dans le cas de l'acide sulfurique.

\section{Ajout du peroxyde d'hydrogène dans la solution réactionnelle}

Des modifications du milieu réactionnel, comme par exemple l'ajout des oxydants $\mathrm{H}_{2} \mathrm{O}_{2}$ ou $\mathrm{S}_{2} \mathrm{O}_{8}{ }^{2-}$, peuvent faire accélérer les processus de photodégradation des composés azoïques. L’influence du peroxyde d'hydrogène sur l'abattement de la concentration des deux composés a fait aussi l'objet de ce travail. Les courbes des abattements de l'absorbance de la solution en fonction $\mathrm{du}$ temps pour différentes concentrations en peroxyde d'hydrogène sont représentées sur la Figure 11. Les résultats obtenus ont montré que, dans le domaine de concentrations choisi, la décoloration dépend de la teneur en peroxyde d'hydrogène. La décoloration totale de la solution de RB5 a été obtenue au bout d'une heure pour la concentration de $3,7 \times 10^{-2}$ mol. $\mathrm{L}^{-1}$ en $\mathrm{H}_{2} \mathrm{O}_{2}$. Un taux de décoloration de $48,5 \%$ a été obtenu au bout d'une heure avec la concentration de $\mathrm{H}_{2} \mathrm{O}_{2}$ de $5,18 \times 10^{-2}$ mol. $\mathrm{L}^{-1}$ dans le cas de RO16. Les rapports de l'absorbance initiale sur l'absorbance de la solution $\operatorname{Abs}_{\mathrm{o}} / \mathrm{Abs}(\mathrm{t})$ obtenus au bout d'un temps de contact de 30 minutes sont représentés en fonction de la concentration en peroxyde d'hydrogène sur la Figure 12. Dans le domaine de concentration étudié, les concentrations les plus favorables pour la décoloration la plus rapide ont été de $3,7 \times 10^{-2} \mathrm{~mol} / \mathrm{L}$ pour $\mathrm{RB} 5$ et $5,18 \times 10^{-2} \mathrm{~mol} / \mathrm{L}$ pour RO16. 


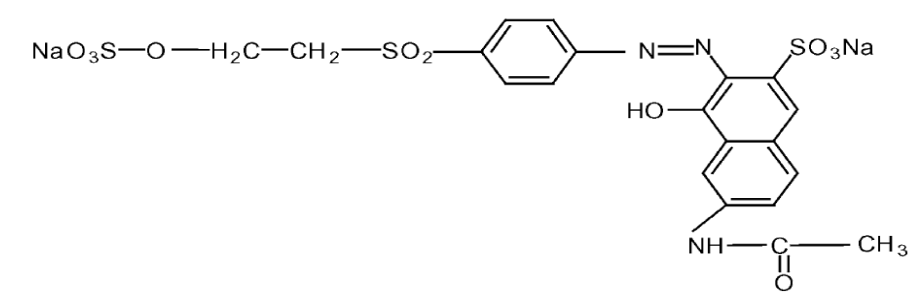

Structure moléculaire du Réactive Orange $16\left(\mathrm{RO} 16, \lambda_{\max }=495 \mathrm{~nm}\right)$

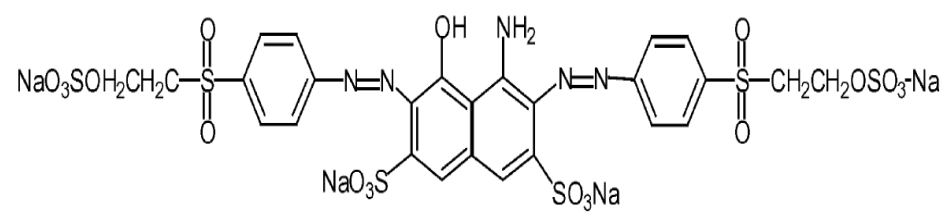

Structure moléculaire du Réactive Black $5\left(\mathrm{RB} 5 \lambda_{\max }=599 \mathrm{~nm}\right)$

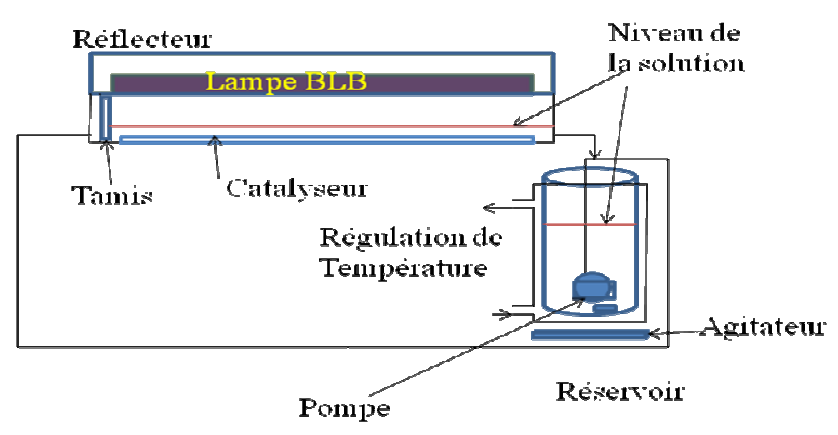

Figure 1 : Schéma du montage expérimental.

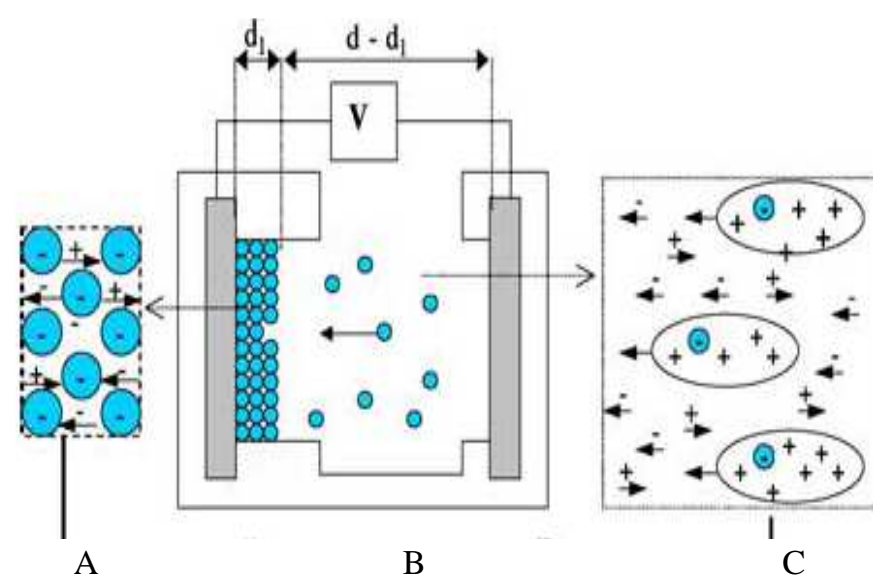

Figure 2: Schéma du montage pour la réalisation de couches minces ou films de $\mathrm{TiO}_{2}$ par le processus d'électrophorèse : (a) coupe de la couche en formation, (b) cuve ou cylindre contenant la suspension, (c) coupe de la suspension montrant le mouvement des particules chargées. 


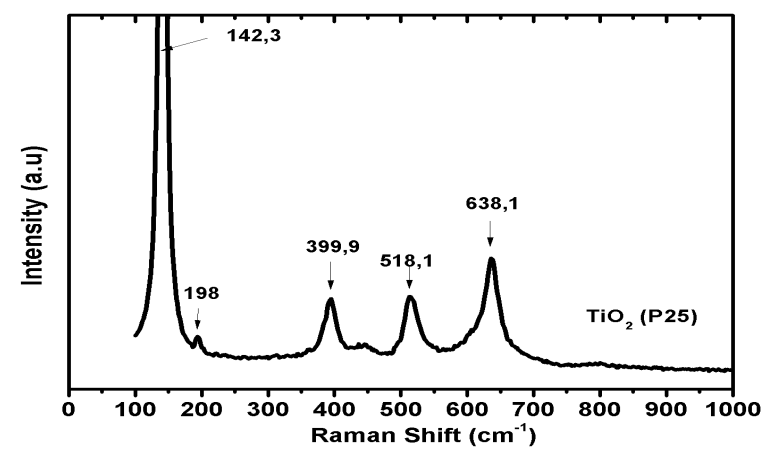

Figure 3: Spectre Raman des couches de $\mathrm{TiO}_{2}(\mathrm{P} 25)$.

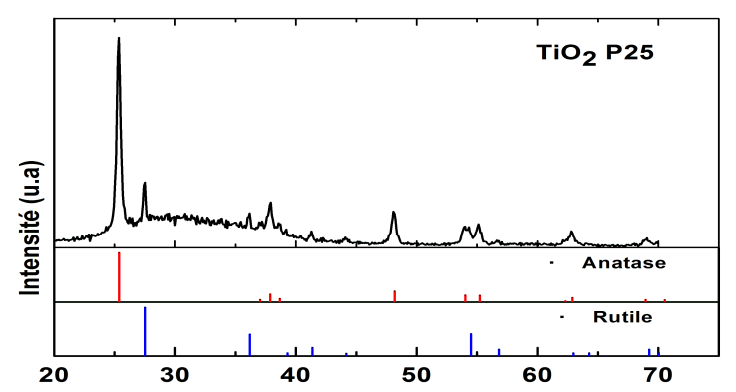

Figure 4 : Spectre de la diffraction des rayons $\mathrm{X}$ des couches de $\mathrm{TiO}_{2}(\mathrm{P} 25)$.
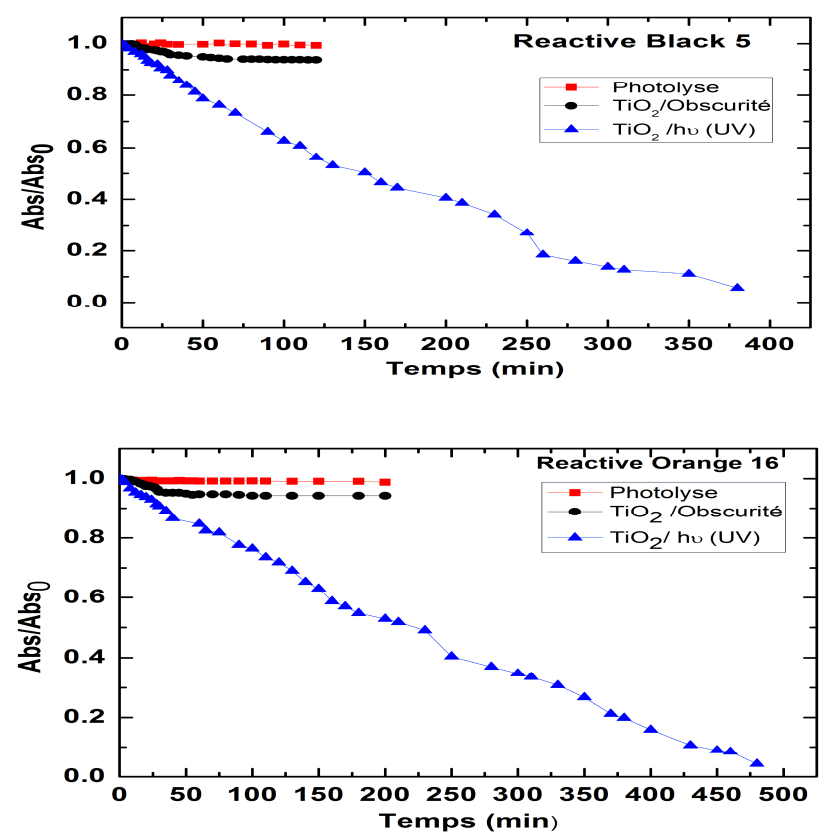

Figure 5 : Cinétique d'élimination de RB5 (5 mgC/L) et de RO16 (5 mgC/L) par photolyse, par adsorption à l'obscurité et par oxydation en présence de la lumière. 


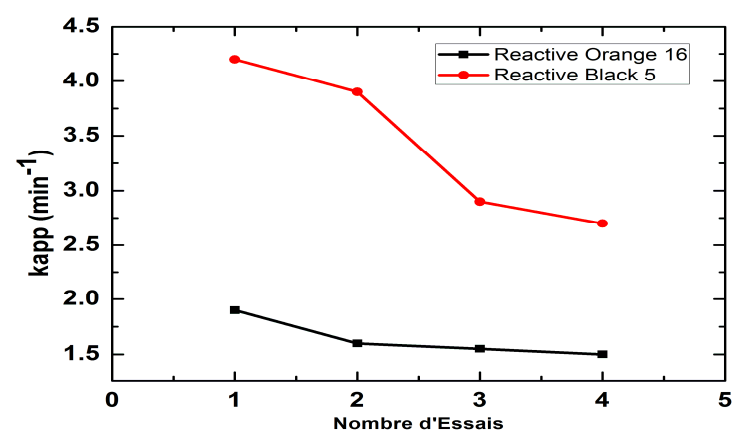

Figure 6: Evolution du $\mathrm{k}_{\mathrm{app}}$ déterminé lors de 4 essais successifs de photodégradation du RB5 et du RO16 réalisés dans les conditions expérimentales indiquées.

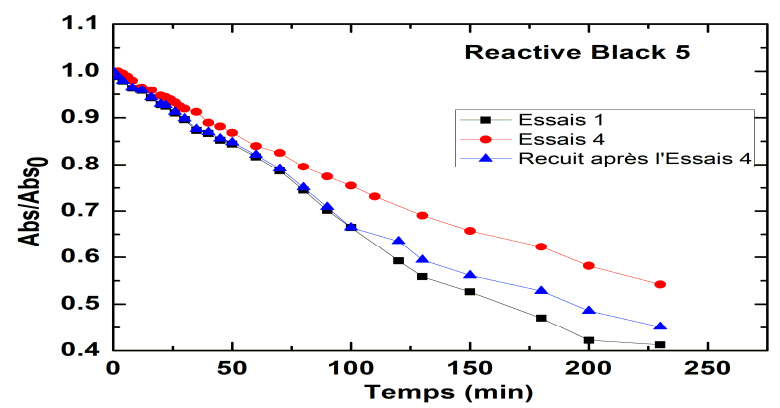

Figure 7 : Réutilisation d'une couche mince de $\mathrm{TiO}_{2}(\mathrm{P} 25)$ Degussa pour la photooxydation du RB5.
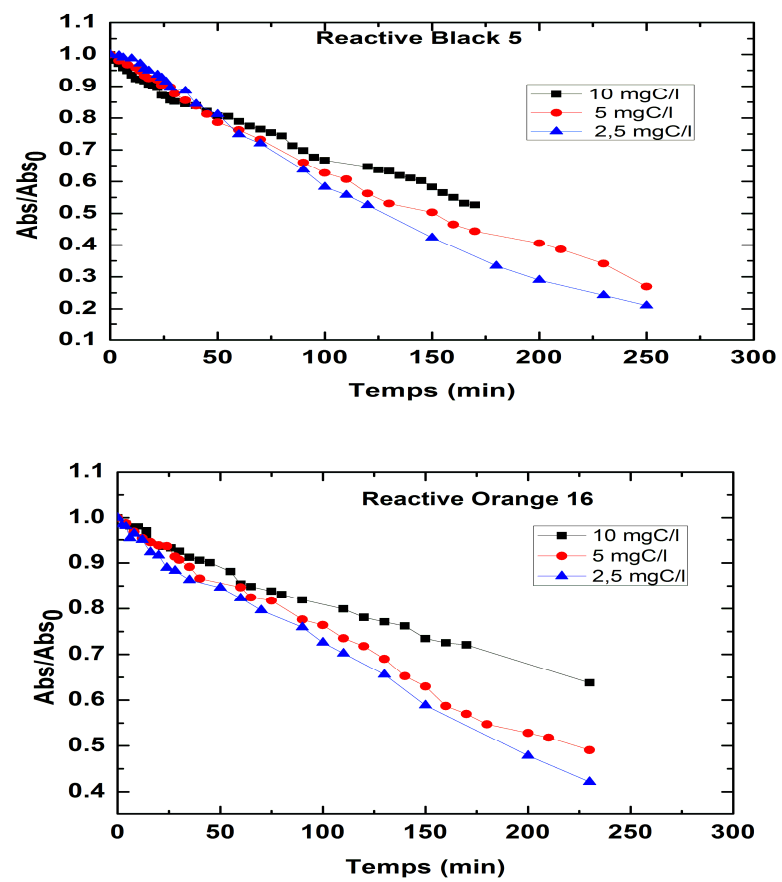

Figure 8 : Influence de la concentration initiale sur la vitesse de dégradation des colorants à pH libre. 

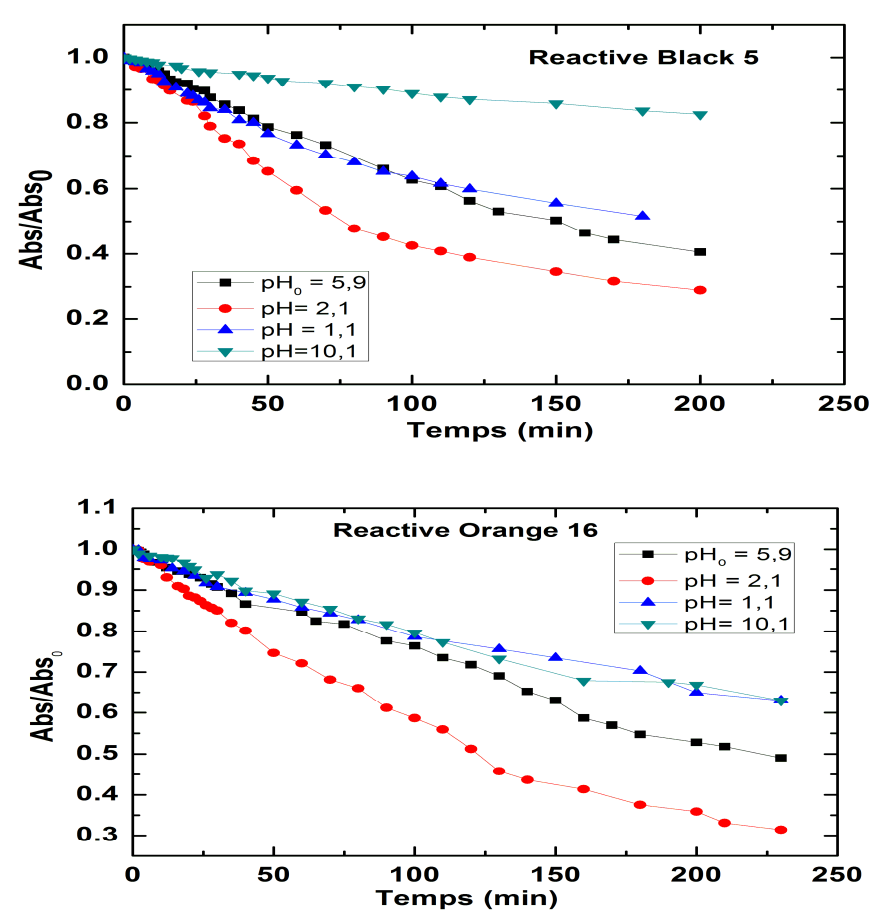

Figure 9: Influence du pH sur la photodégradation de Reactive orange 16 et du Reactive Black 5 de concentration $5 \mathrm{mgC} / \mathrm{L}$.
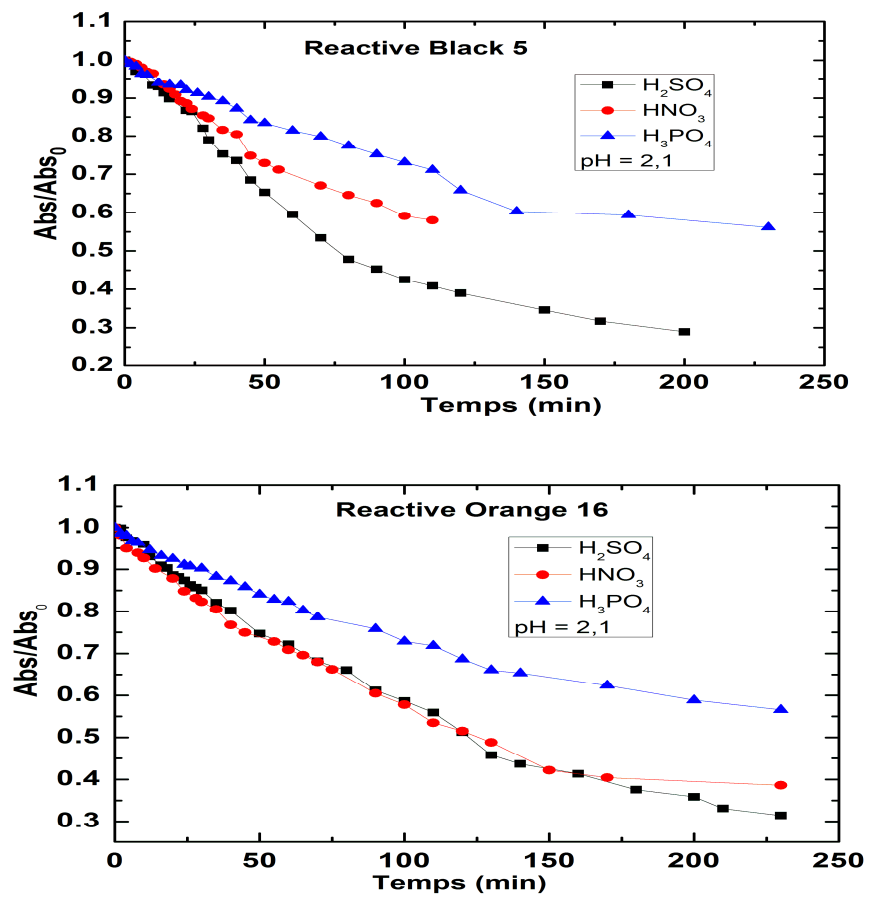


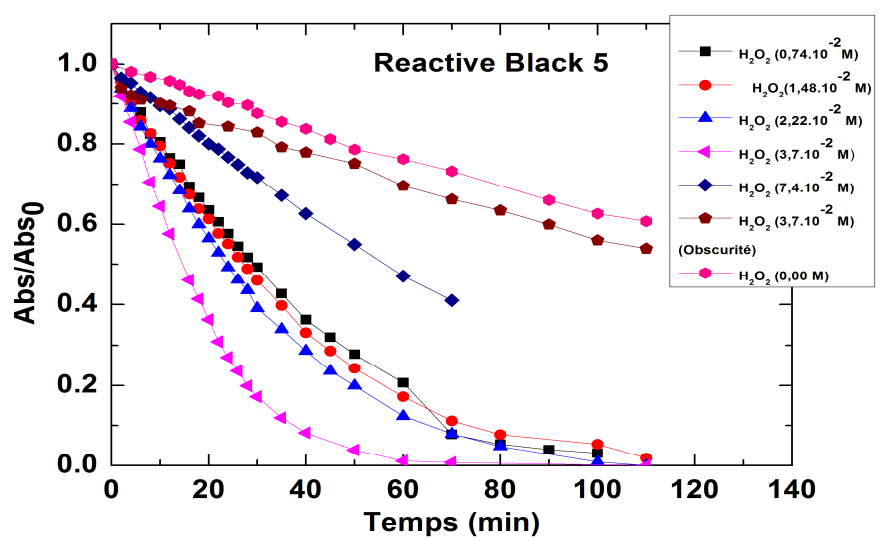

Figure 10 : Influence de la nature de l'acide utilisé pour ajuster le $\mathrm{pH}$ sur la dégradation des colorants.

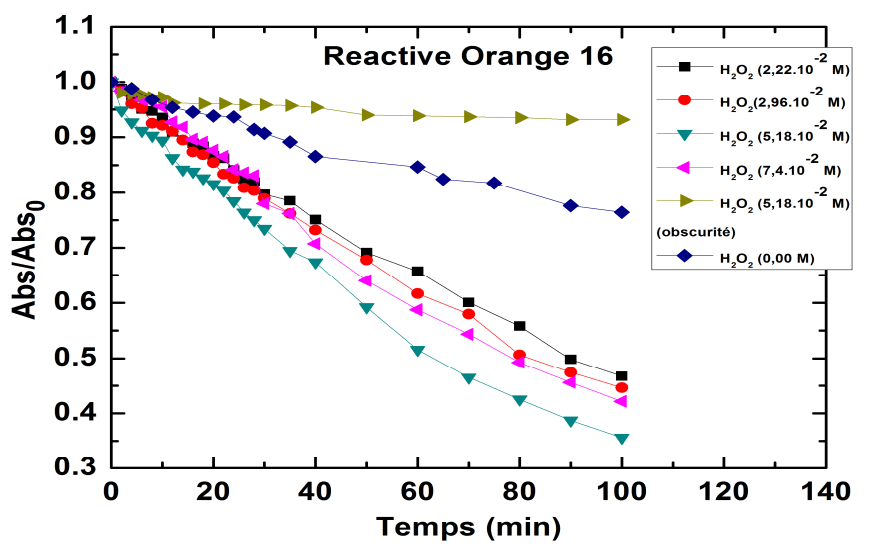

Figure 11: Influence de la concentration initiale en peroxyde d'hydrogène sur la photodégradation des colorants RO16 et RB5 par les couches de $\mathrm{TiO}_{2}(\mathrm{P} 25)$.

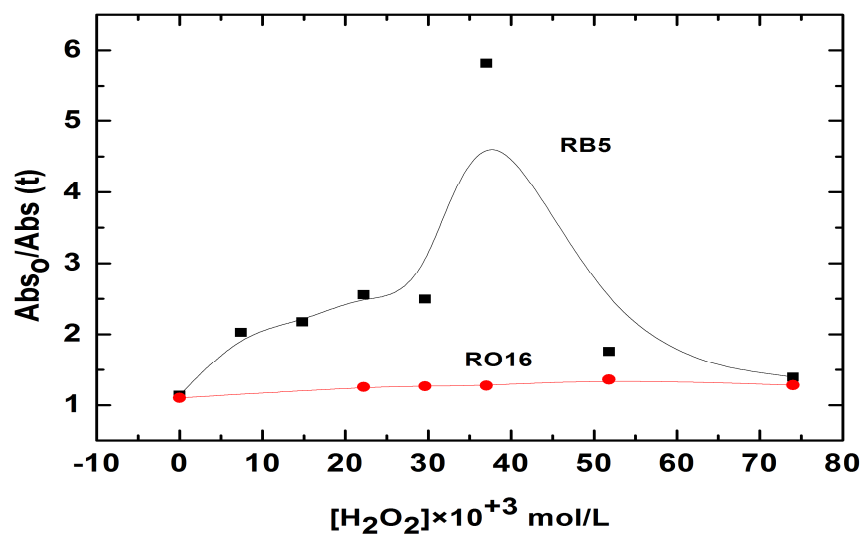

Figure 12: Evolution du rapport $\mathrm{Abs}_{0} / \mathrm{Abs}(\mathrm{t})$ à 30 min en fonction de la concentration initiale en peroxyde d'hydrogène lors de la photodégradation de Reactive Orange 16 et Reactive Black 5. 
Tableau 1 : Taux d'abattement et constante cinétique apparente de vitesse du premier ordre.

\begin{tabular}{|c|c|c|c|c|c|}
\hline & & \multicolumn{2}{|c|}{ Reactive Black 5} & \multicolumn{2}{|c|}{ Reactive Orange 16} \\
\hline & & $\begin{array}{c}\text { Abattement } \\
(\tau \%)\end{array}$ & $\mathbf{k}_{\text {app }}\left(\min ^{-1}\right)$ & $\begin{array}{c}\text { Abattement } \\
(\tau \%)\end{array}$ & $k_{\text {app }}\left(\min ^{-1}\right)$ \\
\hline $\begin{array}{l}\text { Photolyse }(\tau, \%) \\
\text { à } 120 \text { min }\end{array}$ & & 0,7 & - & 0,9 & - \\
\hline $\begin{array}{l}\text { Adsorption }(\tau, \%) \\
\text { à } 120 \mathrm{~min}\end{array}$ & & 6,3 & - & 5,7 & - \\
\hline Photocatalyse $(\tau, \%)$ & $\begin{array}{l}120 \mathrm{~min} \\
380 \mathrm{~min}\end{array}$ & $\begin{array}{l}43,8 \\
94,4\end{array}$ & $4,7 \times 10^{-3}$ & $\begin{array}{l}28,2 \\
80,2\end{array}$ & $\begin{array}{c}- \\
3,1 \times 10^{-3}\end{array}$ \\
\hline
\end{tabular}

Tableau 2 : Constantes apparentes de vitesse et vitesses initiales de dégradation.

\begin{tabular}{ccccc}
\hline & \multicolumn{2}{c}{$\mathbf{k}_{\mathbf{a p p}}\left(\mathbf{m i n}^{-\mathbf{1}}\right) \times \mathbf{1 0}^{\mathbf{+ 3}}$} & \multicolumn{2}{c}{$\left.\mathbf{r}_{\mathbf{0}=} \mathbf{k}_{\mathbf{a p p}} \times \mathbf{C}_{\mathbf{0}} \mathbf{~ m o l} / \mathbf{L} / \mathbf{m i n}\right) \times \mathbf{1 0}^{+\mathbf{6}}$} \\
\cline { 2 - 5 } Concentration $(\mathbf{m g C} / \mathbf{L})$ & $\mathbf{R O 1 6}$ & $\mathbf{R B 5}$ & $\mathbf{R O 1 6}$ & $\mathbf{R B 5}$ \\
\hline 2,5 & 3,4 & 6,4 & 0,072 & 0,094 \\
5 & 3,1 & 4,8 & 0,13 & 0,14 \\
10 & 1,9 & 3,5 & 0,16 & 0,2 \\
\hline
\end{tabular}

Tableau 3 : Constantes apparentes de vitesse pour différentes valeurs de $\mathrm{pH}$.

\begin{tabular}{lcc}
\hline Valeurs de $\mathbf{p H}$ & \multicolumn{2}{c}{$\mathbf{k}_{\text {app }}\left(\mathbf{m i n}^{-\mathbf{1}}\right) \times \mathbf{1 0}^{-\mathbf{3}}$} \\
\cline { 2 - 3 } & $\mathbf{R B 5}$ & $\mathbf{R O 1 6}$ \\
\hline 1,1 & 4,1 & 1,9 \\
2,1 & 8,5 & 5,6 \\
5,9 & 4,7 & 3,1 \\
10 & 0,96 & 2,1 \\
\hline
\end{tabular}

\section{DISCUSSION}

Les pics caractéristiques de la spectroscopie Raman (Figure 3) ont montré que le photocatalyseur est constitué principalement de l'anatase, phase la plus active pour les processus de photocatalyse. Ces résultats confirment ceux obtenus dans la littérature (Colon et al., 2003 ; Li et al., 2007). Mais le spectre DRX du photocatalyseur a montré la présence de l'anatase et du rutile (Figure 4). La présence de ces deux phases dans la poudre mère du $\mathrm{TiO}_{2}(\mathrm{P} 25)$ a été indiquée par le producteur Degusa. Selon ce producteur la poudre mère est constituée de $80 \%$ d'anatase et de $20 \%$ de rutile. En considérant les intensités du pic important du rutile $\left(2 \Theta=27,6^{\circ}\right)$ et celui de l'anatase $(2 \theta=$ $25,4^{\circ}$ ), le rapport de leur intensité a donné 0,218 soit $21,8 \%$ du rutile par rapport à l'anatase. Ces résultats ont montré que ces proportions restent presque identiques dans les couches minces traitées à $450{ }^{\circ} \mathrm{C}$. Au-delà de $450{ }^{\circ} \mathrm{C}$, une transformation allotropique de 
l'anatase en rutile est possible (Chardin et al., 2007).

Dans les conditions opératoires de l'étude, la photolyse et l'adsorption des colorants à l'obscurité n'ont éliminé que des fractions très faibles des colorants RB5 et R016. La quantité de colorant RB5 qui s'est adsorbée sur les couches de $\mathrm{TiO}_{2}$ est légèrement plus importante que celle du colorant RO16. Les constantes apparentes de vitesse de premier ordre ont été déterminées pour une décoloration de $50 \%$. Les taux d'abattement (Tableau 1) ont montré l'efficacité du système photocatalytique à dégrader les colorants textiles étudiés par rapport à la photolyse. La constante de vitesse de la réaction du RB5 a été plus élevée que celle du RO16. La bonne adsorption de RB5 a probablement favorisé sa meilleure dégradation par rapport à celle de RO16. En effet, les réactions de dégradation s'effectuent à l'interface de la solution réactionnelle et du catalyseur. Ainsi, plus les molécules sont adsorbées, plus il y a des possibilités d'échanges pouvant conduire à la réaction de dégradation. L'adsorption des colorants étudiés semble dépendre de la nature et du nombre d'hétéroatomes que portent les molécules de ces colorants. C'est probablement pour cette raison que la quantité de RB5 adsorbée est plus élevée que celle de R016. A la différence, l'exposition des solutions réactionnelles à la lumière et en présence de $\mathrm{TiO}_{2}$ conduit à de très importants abattements de l'absorbance. Cette capacité à dégrader les molécules organiques a été observée lors de récentes études effectuées par Chatterjee et al. (2008) sur les mêmes molécules en utilisant des suspensions aqueuses de $\mathrm{TiO}_{2}(100 \mathrm{mg}$ dans $50 \mathrm{~mL}$ de la solution) soumises à la lumière visible. Ces études ont indiqué un abattement d'environ 95\% après un temps de contact de 11 heures. Les travaux de Zielinska et al. (2001) ont également montré une décoloration totale à la lumière UV-visible au bout de 70 minutes avec une suspension de $0,4 \mathrm{~g} / \mathrm{L}$ de $\mathrm{TiO}_{2}$.
Les valeurs des constantes apparentes de vitesse ont montré que l'activité photocatalytique des couches a diminué en fonction du temps d'utilisation (Figure 6). La baisse de l'activité peut être due à une saturation des sites réactifs du catalyseur par les résidus ou les sous produits de formation des essais précédents. Le traitement à $450{ }^{\circ} \mathrm{C}$ a permis la décomposition de ces résidus probablement en $\mathrm{CO}_{2}, \mathrm{NO}_{2}$ et $\mathrm{SO}_{2}$ libérant les sites et permettant une reprise de l'activité.

En ce qui concerne l'influence de la concentration du colorant, l'augmentation de la vitesse initiale de dégradation s'explique par le fait que plus la concentration du colorant augmente, plus la probabilité de collision entre un radical $\mathrm{HO}^{\circ}$ et une molécule est grande (dégradation indirecte). Cela peut être lié aussi à l'adsorption du colorant qui est plus importante quand la concentration est grande. Dans ce dernier cas, c'est la photocatalyse directe, caractérisée par la dégradation par les trous, qui est favorisée (Villarreal et al., 2004). Avec les concentrations très élevées en colorant, la très forte occupation des sites réactifs du $\mathrm{TiO}_{2}$ et l'opacité de la solution de colorant aux rayonnements utilisés peuvent être la raison pour laquelle il y a une diminution de la vitesse de réaction et par conséquent de la constante apparente de vitesse. Ces observations sont en accord avec d'autres travaux (Sauer et al., 2002 ; Konstantinou et Albanis, 2004; Fangfei et al., 2008 ).

Dans l'étude de l'influence du $\mathrm{pH}$ (Figure 9), la dégradation est plus faible en milieu basique bien que, dans ce milieu, la concentration des ions $\mathrm{OH}^{-}$devrait engendrer une production importante des radicaux $\mathrm{OH}^{\circ}$ liée à une oxydation de ces ions par les trous ( $\mathrm{Xu}$ et al., 2009). En milieu acide, elle est favorisée par une forte adsorption de colorants étudiés par rapport au milieu basique (Konstantinou et Albanis, 2004). En effet, les expériences préliminaires d'adsorption des colorants étudiés ont montré que le taux de molécules retenues sur le $\mathrm{TiO}_{2}$ atteint, au bout d'un temps de contact de 20 minutes, une 
valeur de $10 \%$ au $\mathrm{pH} 2,1$. L'adsorption a été quasi-nulle en milieu basique. De plus, il est connu que la minéralisation des composés organiques en $\mathrm{CO}_{2}$ en milieu fortement basique entraîne une production des carbonates et des bicarbonates reconnus comme étant de véritables pièges à radicaux $\mathrm{OH}^{\circ}$ ( Xu et al., 2009). Avec la solution de $\mathrm{pH}$ 1,1 , une formation d'impuretés sur les couches de $\mathrm{TiO}_{2}$ a été constatée lorsque la couche est gardée quelques heures après la fin des expériences. Ces impuretés sont probablement un produit de l'attaque du substrat d'acier en milieu acide. Elles peuvent agir comme des centres de recombinaison des électrons-trous pouvant entraîner la chute de l'activité du catalyseur. Le taux d'adsorption du colorant est quasi identique avec l'acide nitrique et l'acide sulfurique et a été de l'ordre de $10 \%$ au pH 2,1 au bout de 20 minutes. Par contre avec l'acide phosphorique, un taux d'adsorption de $5 \%$ environ a été obtenu au bout de 20 minutes à l'obscurité. Les ions produits en solution par ces composés minéraux se sont adsorbés sur le $\mathrm{TiO}_{2}$ selon leur forme et ont réduit ainsi la surface active $\mathrm{du}$ catalyseur. Une étude concernant la dégradation de BBP (n-butyle benzyle phthalate) a montré que les anions en solution aqueuse ont un effet inhibiteur non négligeable classé selon l'ordre d'effet néfaste suivant : $\mathrm{PO}_{4}{ }^{3-}>\mathrm{CO}_{3}{ }^{2-}>\mathrm{NO}_{3}{ }^{-}>\mathrm{SO}_{4}{ }^{2-}>\mathrm{Cl}^{-}$ (Xu et al., 2009). Les résultats (Figure 10) obtenus dans cette étude sont donc en accord avec les résultats de Xu et al. (2009) En résumé, la dégradation photocatalytique des composés azoïques a été plus importante au pH acide (Naskar et al., 1998 ; Sakthivel et al., 2003).

Les courbes de la Figue 11 ont montré que le taux d'abattement des deux colorants dépend de la concentration en $\mathrm{H}_{2} \mathrm{O}_{2}$ et que la présence du peroxyde d'hydrogène permet d'améliorer la dégradation des deux colorants. Le peroxyde d'hydrogène réduit la recombinaison des paires électron-trou en piégeant les électrons de la bande de conduction. D'autres transformations de $\mathrm{H}_{2} \mathrm{O}_{2}$ engendrent la production des radicaux $\mathrm{HO}^{\circ}$ (Chen et al., 2003). Les différents mécanismes de décomposition et de production des radicaux hydroxyles peuvent être décrits comme suit (Daneshvar et al., 2003; Konstantinou et Albanis, 2004) :

$\mathrm{H}_{2} \mathrm{O}_{2}+\mathrm{e}_{\mathrm{cb}}{ }^{-} \rightarrow \mathrm{HO}^{\circ}+\mathrm{OH}^{-} ; \mathrm{H}_{2} \mathrm{O}_{2}+\mathrm{hv}$ $\rightarrow 2 \mathrm{HO}^{\circ} ; \mathrm{H}_{2} \mathrm{O}_{2}+\mathrm{H}^{+}+\mathrm{e}^{-} \rightarrow \mathrm{HO}^{\circ}+\mathrm{H}_{2} \mathrm{O}$; $\mathrm{H}_{2} \mathrm{O}_{2}+\mathrm{O}_{2}{ }^{\circ-} \rightarrow \mathrm{HO}^{\circ}+\mathrm{OH}^{-}+\mathrm{O}_{2}$. Il ressort de ces équations que l'ajout du peroxyde d'hydrogène entraine la production supplémentaire des radicaux $\mathrm{HO}^{\circ}$ et par conséquent une augmentation de l'oxydation. Les résultats obtenus ont confirmé ceux existants dans la littérature (So et al., 2002; Saquib et Muneer, 2003; Konstantinou et Albanis, 2004)). Cependant, les concentrations trop élevées ont limité les performances de photooxydation. En effet, Daneshvar et al. (2003) ont reporté que lorsque la concentration devient élevée, le peroxyde d'hydrogène se comporte comme un piège pour les trous de la bande de valence et les radicaux hydroxyles à la surface du catalyseur suivant les réactions:

$\mathrm{H}_{2} \mathrm{O}_{2}+2 \mathrm{~h}_{\mathrm{vb}}+\rightarrow \mathrm{O}_{2}+\mathrm{H}^{+} ; \mathrm{H}_{2} \mathrm{O}_{2}+\mathrm{HO}^{\circ} \rightarrow$ $\mathrm{H}_{2} \mathrm{O}+\mathrm{HO}_{2}^{\circ} ; \mathrm{HO}_{2}^{\circ}+\mathrm{HO}^{\circ} \rightarrow \mathrm{H}_{2} \mathrm{O}+\mathrm{O}_{2}$

Pour chaque colorant, il existe une concentration optimale de $\mathrm{H}_{2} \mathrm{O}_{2}$ pour laquelle la décoloration a été plus importante (Figure 12).

\section{Conclusion}

Cette étude montre qu'il est possible de dégrader par photocatalyse les colorants Reactive Orange 16 et le Reactive Black 5 avec les couches minces de $\mathrm{TiO}_{2}$ (P25) Degussa obtenues par électrophorèse sur de l'acier inoxydable. Ces couches minces subissent, au cours de leur utilisation, un vieillissement par l'occupation des sites actifs par des molécules formées qui entraînent, par conséquent, la baisse de l'activité du photocatalyseur. Un traitement thermique à $450{ }^{\circ} \mathrm{C}$ a permis de libérer partiellement les sites occupés. Les paramètres tels que la concentration initiale du composé organique étudié, le pH initial, l'ajout du peroxyde 
d'hydrogène ont été étudiés. Le suivi de photodégradation des deux colorants a montré que leur dégradation passe par un optimum pour lequel l'abattement du composé est meilleur.

Cette étude montre l'efficacité de la photocatalyse qui peut être exploitée pour la dépollution des eaux issues des usines textiles ou unités artisanales de teinture de pagnes.

\section{REMERCIEMENTS}

Nous remercions l'Agence Universitaire de la Francophonie, la Fondation Internationale pour la Science (IFS) et la Région Poitou-Charentes en France pour leurs financements qui a permis de réaliser ce travail.

\section{RÉFÉRENCES}

Behnajady MA, Modirshahla N. 2006. Kinetic modeling on photooxidative degradation of C.I. Acid Orange 7 in a tubular continuous-flow photoreactor. Chemosphere, 62: 1543-1548.

Chardin T, Coissard V, Berrada P-Ed, Weyer F. 2007. Etudes de surfaces autonettoyantes par photocatalyse, Projet Scientifique collectif : 1- 41.

Chatterjee, Patnam VR, Sikdar A, Joshi P, Misra R, Rao NN. 2008. Kinetics of thedecoloration of reactive dyes over visible light-irradiated $\quad \mathrm{TiO}_{2}$ semiconductor. Photocatalyst. J. Hazard. Mater., 156 : 435-441.

Chen J, Lui M, Zhang L, Zhang J, Jin L. 2003. Application of nano- $\mathrm{TiO}_{2}$ towards polluted water treatment combined with electro-photochemical method. Water Res., 37: 3815-3820.

Colon G, Hidalgo MC, Macias M, Navio JA. 2004. Enhancement of $\mathrm{TiO}_{2} / \mathrm{C}$ photocatalytic activity by sulfate promotion. Appl. Catal. A. General, 259: 235-243.

Colon G, Hidalgo MC, Navio JA. 2003. Photocatalytic behaviour of sulphated $\mathrm{TiO}_{2}$ for phenol degradation. Appl. Cata. B. Environ, 45: 39-50.
Daneshvar N, Salari D, Khataee AR. 2003. Photocatalytic degradation of azo dye acid red 14 in water: investigation of the effect of operational parameters. $J$. Photochem. Photobiol. A. Chem., 157: 111-116.

El Hajjouji H, Barje F, Pinelli E, Bailly J-R, Richard C, Winterton P, Revel J-C, Hafidi M. 2008. Photochemical UV/TiO 2 treatment of olive mill wastewater (OMW). Bioresour. Technol., 99: 72647269.

Galindo C, Jacques P, Kalt A. 2001. Photooxidation of the phynelazonaphtol AO20 on $\mathrm{TiO}_{2}$ : Kinetics and mechanistic investigations. Chemosphere, 45: 997.

Galindo C, Jacques P, Kalt A. 2000. Photodegradation of the aminoazobenzene acid orange 52 by three advanced oxidation processes: $\mathrm{UV} / \mathrm{H}_{2} \mathrm{O}_{2}$, $\mathrm{UV} / \mathrm{TiO}_{2}$ and $\mathrm{VIS} / \mathrm{TiO}_{2}$ Comparative mechanistic and kinetic investigations. $J$. Photochem. Photobiol. A. Chem., 130: 35- 47.

Houas A, Lachheb H, Ksibi M, Elaloui E, Guillard C, Hermann JM. 2001. Photocatalytic degradation pathway of methylene blue in water. Applied Catalysis B. Environmental, 31: 145-157.

Li J-G, Ishigaki T, Sun X. 2007. Anatase, Brookite, and Rutile Nanocrystals via Redox Reactions under Mild Hydrothermal Conditions: PhaseSelective Synthesis and Physicochemical Properties. J. Phys. Chem. C., 111: 49694976.

Li F, Sun S, Jiang Y, Xia M, Sun M, Xue B. 2008. Photodegradation of an azo dye using immobilized nanoparticles of $\mathrm{TiO}_{2}$ supported by natural porous mineral. $J$. Hazard. Mater., 152: 1037-1044.

Konstantinou IK, Albanis TA. 2004. $\mathrm{TiO}_{2}-$ assisted photocatalytic degradation of azo dyes in aqueous solution: kinetic and mechanistic investigations. A review. Applied Catalysis B. Environmental, 49: 1-14. 
Naskar S, Arumugam S, Pillay, Chanda M. 1998. Photocatalytic degradation of organics dyes in aqueous solution with $\mathrm{TiO}_{2}$ nanoparticles immobilized on foamed polyethylene sheet. $J$. Photochem. Photobiol. A. Chem, 113: 257-264.

Pedroza AM, Mosqueda R, Alonso-Vante N, Rodríguez-Vázquez R. 2007. Sequential treatment via Trametes versicolor and $\mathrm{UV} / \mathrm{TiO}_{2} / \mathrm{Ru}_{x} \mathrm{Se}_{y}$ to reduce contaminants in waste water resulting from the bleaching process during paper production. Chemosphere, 67: 793-801.

Piscopo A. 2002. Chimie solaire et traitement photocatalytique des eaux polluées:applications aux traitements sélectifs et exemples d'utilisation de catalyseurs supportés, Thèse de doctorat, Université de Metz.

Saquib M, Muneer M. 2003. $\mathrm{TiO}_{2}$-mediated photocatalytic degradation of a triphenylmethane dye (gentian violet), in aqueous suspensions. Dyes Pigments, 56: $37-49$.

Sakthivel S, Meppolian B, Shankar MV, Arabindoo B, Palanichanmy M, Murugesan V. 2003. Solar photocatalytic degradation of azo dye: comparison of photocatalytic efficiency of $\mathrm{ZnO}$ and $\mathrm{TiO}_{2}$. Sol. Energy Mater. Sol. Cells, 77: 65.

Sauer T, Neto CG, José HJ, Moreira FPM. 2002. Kinetics of photocatalytic degradation of reactive dyes in a $\mathrm{TiO}_{2}$ slurry reactor. J. Photochem. Photobiol. A. Chem., 149: 147-154.

Shukla SS, Dorris KL, Chikkaveeraiah B. 2009. Photocatalytic degradation of 2,4dinitrophenol. J. Hazard Mater., 164: 310-314.

Slokar YM, Le Marechal AM. 1998. Methods of Decoloration of Textile Waste water. Dyes Pigments, 37: 335.
So CM, Cheng MY, Yu JC, Wong PK. 2002. Degradation of azo dye Procion Red MX-5B by photocatalytic oxidation. Chemosphere, 46: 905.

Villarreal TL, Bogdanoff P, Salvador P, Alonso-Vante N. 2004. Photocatalytic oxidation on nanostructured chalcogenide modified titanium dioxide. Sol. Energy Mater. Sol. Cells, 83: 347-362.

Wong YC, Szeto YS, Cheung WH, McKay G. 2004. Adsorption of acid dyes on chitosan-equilibrium isotherm analyses. Process Biochem., 39: 693-702.

Xu X-R, Li S-X, Li X-Y, Gu Ji-D, Chen F, Li X-Z, Li H-B. 2009. Degradation of n-butyl benzyl phthalate using $\mathrm{TiO}_{2} /$ UV. J. Hazard. Mater., 164: $527-$ 532.

Zainal Z, Hui LK, Hussin MZ, Abdullah AH, Hamadnech IR. 2009. Characterization of $\mathrm{TiO}_{2}$ Chitosan/Glass photocatalyst for the removal of a monoazo dye via Photodegradation-adsorption process. J. Hazard Mater, 164: 138-145.

Zhu Y, Zhang L, Wang L, Fu Y, Cao L. 2001. The preparation and chemical structure of $\mathrm{TiO}_{2}$ film photocatalysts supported on stainless steel substrates via the sol-gel method. J. Mater. Chem., 11: 1864-1868.

Zielinska B, Grzechulska J, Grzmil B, Morawski AW. 2001. Photocatalytic degradation of Reactive Black 5. A comparison between $\mathrm{TiO}_{2}$-Tytanpol A11 and $\mathrm{TiO}_{2}$-Degussa P25 photocatalyst. Appl. Catal. B. Environ, 35: L1-L7. 